\title{
Polarized ATR-FTIR Spectroscopy of the Membrane-Embedded Domains of the Particulate Methane Monooxygenase ${ }^{\dagger}$
}

\author{
Madhuri S. Vinchurkar, ${ }^{\ddagger}$ Kelvin H.-C. Chen,${ }^{\ddagger}$ Steve S.-F. Yu, ${ }^{\ddagger}$ Shan-Jen Kuo, ${ }^{\ddagger}$ Hui-Chi Chiu, ${ }^{\ddagger}$

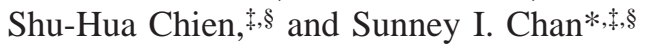 \\ Institute of Chemistry, Academia Sinica, Nankang, Taipei, 11529 Taiwan, and \\ Department of Chemistry, National Taiwan University, Taipei, 10764 Taiwan
}

Received May 15, 2004; Revised Manuscript Received August 18, 2004

\begin{abstract}
The particulate methane monooxygenase (pMMO) of Methylococcus capsulatus (Bath) is an integral membrane protein that catalyzes the conversion of methane to methanol. To gain some insight into the structure-reactivity pattern of this protein, we have applied attenuated total reflection Fourier transform infrared (ATR-FTIR) spectroscopy to investigate the secondary structure of the pMMO. The results showed that ca. $60 \%$ of the amino acid residues were structured as $\alpha$-helices. About $80 \%$ of the peptide residues were estimated to be protected from the amide ${ }^{1} \mathrm{H} /{ }^{2} \mathrm{H}$ exchange during a $21 \mathrm{~h}$ exposure to ${ }^{2} \mathrm{H}_{2} \mathrm{O}$. In addition, a significant portion of the protein was shown to be sequestered within the bilayer membrane, protected from trypsin proteolysis. The ATR-FTIR difference spectrum between the intact and the proteolyzed pMMO-enriched membranes revealed absorption peaks only in the spectral regions characteristic for unordered and $\beta$-structures. These observations were corroborated by amino acid sequence analysis of the pMMO subunits using the program TransMembrane topology with a Hidden Markov Model: 15 putative transmembrane $\alpha$-helices were predicted. Finally, an attempt was also made to model the three-dimensional folding of the protein subunits from the sequence using the Protein Fold Recognition Server based on the 3D Position Specific Scoring Matrix Method. The C-terminal solvent-exposed sequence (N255-M414) of the pMMO $45 \mathrm{kDa}$ subunit was shown to match the $\beta$-sheet structure of the multidomain cupredoxins. We conclude on the basis of this ATR-FTIR study that pMMO is an $\alpha$-helical bundle with ca. 15 transmembrane $\alpha$-helices embedded in the bilayer membrane, together with a water-exposed domain comprised mostly of $\beta$-sheet structures similar to the cupredoxins.
\end{abstract}

Methanotrophic bacteria consume methane produced in anaerobic sediments and thus limit its flux to the atmosphere, where it acts as a greenhouse gas $(1,2)$. The enzyme methane monooxygenase $(\mathrm{MMO})^{1}$ present in these bacteria catalyzes the conversion of methane to methanol using dioxygen as a cosubstrate at ambient pressures and temperatures $(3,4)$. At present, methane is underutilized as an energy source because of the lack of efficient processes for its conversion to the liquid form. The MMO system would provide an ideal natural model to study methane activation and functionalization, a subject of significant current interest (5).

† This work was supported by grants from the National Science Council of the Republic of China (Grant NSC 90-2113-M-001-006, 90-2113-M-001-080, 91-2113-M-006-006, 91-2113-M-001-045, and 922113-M-001-057).

* To whom correspondence should be addressed. Tel: 886-2-27898654. Fax: 886-2-2783-1237. E-mail: chans@chem.sinica.edu.tw.

Academia Sinica.

$\S$ National Taiwan University.

${ }^{1}$ Abbreviations: MMO, methane monooxygenase; pMMO, particulate methane monooxygenase; sMMO, soluble methane monooxygenase; ATR-FTIR, attenuated total reflection Fourier transform infrared; IRE, internal reflection element; DTGS, deuterated triglycine sulfate; MCT, mercury-cadmium-tellurium; ESI-TOF-MS, electrospray ionization time-of-flight mass spectrometry; EPR, electron paramagnetic resonance; SDS-PAGE, sodium dodecyl sulfate-polyacrylamide gel electrophoresis; PIPES, 1,4-piperazine bis(ethane-sulfonic acid, sodium salt); fwhh, full width at half-height; DMPC, dimyristoylphosphatidylcholine.
Two distinct forms of MMOs are known to exist at different cellular locations in methanotrophs, a cytoplasmic soluble form (sMMO) and a membrane-bound particulate form (pMMO) (6). Apart from their biological functions and ecological importance, the unique ability of MMOs to oxidize a broad range of hydrocarbons in addition to methane has garnered increased attention (7-10). Several research groups have studied the sMMO extensively $(11-13)$. The X-ray crystal structures of the sMMO hydroxylase isolated from Methylococcus capsulatus (Bath) and Methylosinus trichosporium OB3b have been solved $(14,15)$. The hydroxylase active site of sMMO contains a nonheme binuclear iron cluster. In contrast, a recent study from this laboratory indicates that the pMMO from $M$. capsulatus is a novel multiple-copper-containing enzyme $(16-20)$. However, as a membrane protein, it has been difficult to define the location and spatial arrangement of the various metallic cofactors of the pMMO, not to mention the three-dimensional folding of the polypeptides in the lipid bilayer.

It is well-known that numerous experimental obstacles are encountered in the determination of the three-dimensional structures of membrane proteins, whether by NMR or diffraction methods. Considering the paucity of structural data, any structural insight gained from other biophysical methods is therefore particularly useful for membrane proteins. Polarized attenuated total reflection Fourier trans- 
form infrared spectroscopy (ATR-FTIR) is currently one of such techniques widely used in membrane protein structural analysis $(21-27)$. This method yields information about the polypeptide secondary structure as well as its orientation in the membrane. The infrared vibrational frequencies can be used to determine the protein secondary structure, while the orientation is obtained from the observed dichroism.

An additional strength of this spectroscopy is that it allows simultaneous study of the structure of lipids and proteins in intact biological membranes. Taking advantage of this unique property, we have applied this technique to generate distinct IR spectra of the membrane-embedded pMMO. We report here how the use of this method in combination with proteolysis and ${ }^{1} \mathrm{H} /{ }^{2} \mathrm{H}$ exchange has enabled us to gain the first experimental glimpse of the structure and dynamics of the transmembrane domain of pMMO.

\section{MATERIALS AND METHODS}

Sample Preparation. The growth of methanotrophs, isolation of pMMO-enriched membranes, and pMMO activity assays were essentially the same as described elsewhere (19). In our laboratory, we have devised a reproducible procedure for the preparation of high quality pMMO from M. capsulatus (Bath) in high yields in a Bioflo 3000 fermentor that is adapted with a hollow fiber membrane bioreactor. Under the standardized growth conditions, pMMO comprises more than $80 \%$ of the total membrane proteins. pMMO-enriched membranes as isolated were used during all of the spectral measurements without further purification, except where noted otherwise. As the pMMO in these membranes has been exposed to air for prolonged periods without turnover in the presence of methane or other hydrocarbon substrates, the pMMO-enriched membranes thus isolated have been referred to as "as-isolated" pMMO membranes (19). Under these conditions, the copper ions in the pMMO in these membranes have become oxidized to the fullest extent possible under these conditions (vide infra).

Growth of Methanotrophs. M. capsulatus (Bath) (ATCC 33009) were maintained on Petri plates containing the NMS medium (ATCC: 1306 nitrate mineral salts medium) and solidified with $1.7 \%$ agar. Cell cultures were maintained under an atmosphere of $20 \%$ methane in air and streaked onto fresh plates every 4-6 weeks. The organisms were first transferred from Petri plates to $250 \mathrm{~mL}$ flasks and subsequently to $2 \mathrm{~L}$ Erlenmeyer flasks containing, respectively, 30 and $300 \mathrm{~mL}$ of NMS medium in $20 \%$ methane in air atmosphere. After $48 \mathrm{~h}$ of incubation with continual shaking, the resulting cell suspension was used to seed a Bioflo 3000 fermentor with a $5 \mathrm{~L}$ vessel containing $3 \mathrm{~L}$ of NMS medium. When the turbidity of the culturing medium had reached $\mathrm{OD}_{595} 1.2-1.6$, typically after a period of $24 \mathrm{~h}$ of incubation, additional NMS medium was added to increase the total culture volume to $5 \mathrm{~L}$ for further semicontinuous growth. It was usually required to replenish the cell-enriched medium with fresh NMS buffer by draining away the used medium in the bioreactor $2-3$ times every $12-24 \mathrm{~h}$. This media replenishment would keep the cell cycle in the middle-log phase to late-log phase over the said period. With $M$. capsulatus (Bath), it was possible to keep the cells growing at $\mathrm{pH} 6.8-7.4$ for $12-24 \mathrm{~h}$ by agitating the cellular suspension in the range of $200-800 \mathrm{rpm}$ and controlling the methane- and air-feeding rate $(0.7-1.3 \mathrm{~L} / \mathrm{min})$ to maintain the dissolved oxygen content to $2.5 \%$ of saturation.

After the cells had been cultured at $30 \mu \mathrm{M}$ copper concentration for $12-24 \mathrm{~h}$ and were ready for harvest, about $4 \mathrm{~L}$ of the medium was removed with the assistance of the preequipped hollow fiber filter. Three-quarters of the resulting concentrated cell suspension were drained out and centrifuged, and the harvested pellet was retained for further characterization. The remainder of the concentrated cell suspension was then filtered to almost dryness. These cells were resuspended in the fermentor with $5 \mathrm{~L}$ of fresh NMS medium injected through the inlet tube into the hollow fiber filter for the next round of culturing. Typically, about 35$40 \mathrm{~g}$ of cells were harvested during the above medium renewal process.

Isolation of pMMO-Enriched Membranes. The harvested cell paste was suspended in $25 \mathrm{mM}$ 1,4-piperazine bis(ethanesulfonic acid, sodium salt) (PIPES) buffer at $\mathrm{pH} 7.0$ (1.2 $\mathrm{mL}$ of buffer/g of cell paste) containing $0.01 \mathrm{mg} / \mathrm{mL}$ of DNase I. Cell suspensions were passed three times through a French pressure cell at 20000 psi in order to separate the cytosolic and membrane fractions from the cell wall. Unlysed cells and cell debris were removed by centrifugation at $27000 \mathrm{~g}$ for $20 \mathrm{~min}$. The supernatant was then ultracentrifuged at $220000 \mathrm{~g}$ for $40 \mathrm{~min}$ to pellet the membrane fraction. The clear supernatant obtained after ultracentrifigation was used as the cytosolic fraction. The pelleted membranes often showed distinct layers. The minor bluish-black bottom layer and the thin white top layer were discarded. Only the middle cut, or the translucent intracytoplasmic membrane fraction, which constituted the bulk of the membrane proteins, was collected. The translucent membranes were washed by suspending them in washing buffer containing $25 \mathrm{mM}$ PIPES, $5 \mathrm{mM}$ ascorbate, and $25 \mu \mathrm{g} / \mathrm{mL}$ of catalase (pH 7.0) using a Dounce homogenizer, repelleted by ultracentrifugation, and resuspended in washing buffer of 2-3 times the volume of the original cell suspension. This process was repeated two or three times until the supernatant was virtually free of soluble proteins. The membranes contained $\sim 40 \mathrm{mg} / \mathrm{mL}$ pMMO as determined by the Lowry method, and the proteinto-lipid ratio was found to be ca. 4:1 (w:w). Membrane lipid isolation, protein purification, and reconstitution were carried out as described elsewhere $(7,18,19)$.

Proteolysis of pMMO in the pMMO-Enriched Membranes. pMMO-enriched membranes were subjected to trypsin digestion in order to separate the solvent-exposed and membranebound domains of the polypeptides (19). A solution mixture of pMMO-enriched membranes and trypsin (100:1) in 25 $\mathrm{mM}$ ammonium bicarbonate buffer $(\mathrm{pH} 8)$ was incubated in a temperature-controlled water bath at $37^{\circ} \mathrm{C}$ for $3 \mathrm{~h}$. After incubation, the solution was centrifuged at $10000 \mathrm{~g}$ for 15 min to separate the soluble and membrane fractions. The membrane fraction was washed 2-3 times by deionized water prior to the ATR-FTIR measurements.

The lyophilized soluble fraction containing the tryptic peptides of the pMMO was dissolved in deionized water and treated with $\mathrm{C} 18$ ZipTip. The tryptic peptides were then analyzed by peptide mass fingerprinting by using liquid chromatography nano-flow electronspray ionization (ESI) equipped with tandem mass (MS/MS) in a QSTAR pulsar quadrupole time-of-flight (TOF) mass spectrometer. Monoisotopic mass values were used to search the possible amino 
acid composition of the peptides based on the NCBI database using the MS-Fit search engine (http://prospector.ucsf.edu). The tolerance for the input mass was set at $100 \mathrm{ppm}$, and the number of missed cleavages was set from 0 to 1 .

Preparation of Fully Reduced and Fully Oxidized pMMO in pMMO-Enriched Membranes. Fully reduced and fully oxidized pMMO from the as-isolated pMMO membranes were prepared according to procedures established in this laboratory (28). The as-isolated pMMO membranes were first purged with dioxygen-free argon. The membrane solution was then mixed with $40 \mathrm{mM}$ sodium dithionite solution to obtain fully reduced protein. The amount of reductant required to fully reduce the protein was judged from the "null" electron paramagnetic resonance (EPR) signal. To obtain fully oxidized protein, the fully reduced protein was titrated with $100 \mathrm{mM}$ potassium ferricyanide solution. The extent of oxidation of the protein was determined from the copper EPR signal of the ferrocyanide-pMMO adduct ( 7 , 28 ), and the enzyme was judged to be fully oxidized when there was no further increase in the EPR intensity. Prior to reduction or oxidation of the pMMO-enriched membranes, the PIPES buffer (25 mM, pH 7.4), the reductant sodium dithionite, and the oxidant potassium ferricyanide were deoxygenated by successive freezing/vacuum/dioxygen-free argon cycles for $10 \mathrm{~min}$.

ATR-FTIR Measurements. ATR-FTIR spectra were recorded at room temperature on a BOMEM-DA8-FTIR spectrophotometer equipped with a deuterated triglycine sulfate (DTGS) or a mercury-cadmium-tellurium (MCT) detector at a resolution of $1 \mathrm{~cm}^{-1}$. A total of 100 scans were averaged for each spectral measurement. The internal reflection element (IRE) was a germanium prism plate with an aperture angle of $45^{\circ}$. Two sample films of oriented multilayers were obtained by slowly evaporating $30 \mu \mathrm{L}$ dispersions of the membrane $[1 \mathrm{mg} / \mathrm{mL}$ (thin film) or 40 $\mathrm{mg} / \mathrm{mL}$ (thick film)] in PIPES buffer ( $25 \mathrm{mM}, \mathrm{pH} 7.2$ ) on one side of the IRE under a stream of dry nitrogen (29). In this manner, thin and thick films of the multilayer sample corresponding to thicknesses of $\sim 0.08$ and $\sim 4.3 \mu \mathrm{m}$, respectively, were obtained. The thickness of the sample film was estimated from the amount of membranes deposited over the Ge IRE and the surface area $\left(3.46 \mathrm{~cm}^{2}\right)$ of the IRE. The IRE was kept under vacuum in a universal sample holder during the measurements. The spectrophotometer was purged with dry air except during oxidation-reduction experiments where dry nitrogen was used.

Secondary Structure Determination. The spectral window that exists between 1700 and $1500 \mathrm{~cm}^{-1}$ is the fingerprint region for the protein secondary structure. The most intense absorption band observed in the region $1700-1600 \mathrm{~cm}^{-1}$ is the amide $\mathrm{I}$ band due to $v(\mathrm{C}=\mathrm{O})$ and some in-plane $\mathrm{N}-\mathrm{H}$ bending. The amide II peak occurs in the $1580-1510 \mathrm{~cm}^{-1}$ region, mainly arising from the in-plane $\mathrm{N}-\mathrm{H}$ bending and $v(\mathrm{C}-\mathrm{N})$. Secondary structure determination is based on the conformation sensitive shape of the amide I band (30-33). The frequency limits for different types of secondary structures in proteins in $\mathrm{H}_{2} \mathrm{O}$ environment, contributing to the amide I band, are well-documented $(27,34,35)$ and are assigned as follows: $1658-1650 \mathrm{~cm}^{-1}, \alpha_{\mathrm{I}}$-helix; $1666-1658$ $\mathrm{cm}^{-1}, \alpha_{\mathrm{II}}$-helix; $1670-1660 \mathrm{~cm}^{-1}, 3_{10}$-helix; 1638-1632 $\mathrm{cm}^{-1} ; 1695-1675 \mathrm{~cm}^{-1}$, antiparallel $\beta$-sheet; $1625-1615$ $\mathrm{cm}^{-1}$, intermolecular $\beta$-sheet; $1685-1655 \mathrm{~cm}^{-1}, \beta$-turns;
$1690-1650 \mathrm{~cm}^{-1}, \gamma$-turns; $1645-1638 \mathrm{~cm}^{-1}$, random coil; and $1618-1605 \mathrm{~cm}^{-1}$, amide or aromatic side chains.

${ }^{1} \mathrm{H} / 2 \mathrm{H}$ Exchange. After the ATR-FTIR spectrum of the thin pMMO film was recorded, the IRE was kept in an evacuated desiccator with $\mathrm{P}_{2} \mathrm{O}_{5}$ for dehydration of the protein film. The spectrum for the dehydrated $(21 \mathrm{~h}) \mathrm{pMMO}$ membrane film was recorded. This dehydrated pMMO membrane film was then rehydrated with ${ }^{2} \mathrm{H}_{2} \mathrm{O}$ by adding ${ }^{2} \mathrm{H}_{2} \mathrm{O}$ over the surface to wet the film. The IRE with this deuterated sample film was kept in a sealed desiccator with ${ }^{2} \mathrm{H}_{2} \mathrm{O}$-saturated $\mathrm{K}_{2} \mathrm{SO}_{4}$ for $21 \mathrm{~h}$. The spectrum for the ${ }^{2} \mathrm{H}_{2} \mathrm{O}$-saturated pMMO film was recorded to monitor the ${ }^{1} \mathrm{H} /{ }^{2} \mathrm{H}$ exchange.

Secondary Structure Orientation from Polarized ATRFTIR. For a molecule fluctuating axially symmetrically about the membrane normal, a molecular order parameter can be defined, which is a function of the amplitude of the timeand spatial-averaged fluctuations around the membrane normal. The order parameter of an $\alpha$-helical peptide in a supported lipid bilayer can be derived from the measured dichroic ratio $\left(R^{\text {ATR }}\right)$ in the spectral region $1662-1645 \mathrm{~cm}^{-1}$ $(36-38)$. The dichroic ratio is defined as the ratio between the absorption of light polarized parallel and perpendicular to the surface of the IRE.

For the $\alpha$-helices of pMMO, the $R^{\text {ATR }}$ was determined using the integrated peak areas of the amide I band in the region 1662-1645 $\mathrm{cm}^{-1}$. Before the area calculations, a straight baseline passing through the ordinates at 1700 and $1600 \mathrm{~cm}^{-1}$ was subtracted. This method of correcting for the contribution of the baseline to the amide I integrated peak areas seems reasonable since no significant protein absorption occurs at these designated wavelengths. This ratio was then used to calculate the molecular order parameter $\left(S_{\text {molecule }}\right)$ of the proteins as follows:

$$
\begin{aligned}
& S_{\text {molecule }}= \\
& \quad\left(E_{x}^{2}-R^{\mathrm{ATR}} E_{y}^{2}+E_{z}^{2}\right) /\left(E_{x}^{2}-R^{\mathrm{ATR}} E_{y}^{2}-2 E_{z}^{2}\right)
\end{aligned}
$$

where $E_{x}, E_{y}$, and $E_{z}$ denote the normalized electric field components when the IR beam is polarized in the respective dimensions.

The values of $E_{x}, E_{y}$, and $E_{z}$ depend on whether the thickness of the deposited film is greater or smaller as compared with the penetration length of the evanescent wave in the ATR-FTIR experiment. The decay depth of the evanescent wave is typically of the order of $0.5 \mu \mathrm{m}$. At the wavelength of the amide I absorption, the penetration depth is $0.4 \mu \mathrm{m}$, which extends far beyond the thickness of our thin deposited film $(\sim 0.08 \mu \mathrm{m})$, and $E_{x}=1.403, E_{y}=1.5$, and $E_{z}=1.375$ for the sample configuration used in our measurements: angle of incidence of IR $\left(\theta=45^{\circ}\right)$; refractive indices of the IRE ( $n=4$ for Ge); refractive index of the sample $(n=1.45$ for protein; 1.43 for lipid bilayer; and 1.33 for water) (27). If the sample is much thicker than the penetration depth of the evanescent wave (the thickness of our thick sample film is $\sim 4.3 \mu \mathrm{m}$ ), then the electric field components are independent of the refractive index of the medium beyond the surface film, and $E_{x}=1.398, E_{y}=$ 1.516 , and $E_{z}=1.625$ (39).

The molecular order parameters for the proteins and lipids could be determined for both the thin or the thick samples from the measured ATR dichroic ratios by using eq 1 . For 
a set of axially symmetric distributions of protein molecules, the order parameter $(S)$ can be written as

$$
S_{\text {proteins }}=S_{\text {helix }} \cdot S_{\text {dipole }}
$$

where $S_{\text {helix }}$ is the order parameter describing the orientations of the helices within the membrane plane about the membrane normal and $S_{\text {dipole }}$ is the order parameter describing the distribution of dipole orientations sampled in the polarized spectrum with respect to the transmembrane helix axis (molecular director). $S_{\text {helix }}$ and $S_{\text {dipole }}$ are calculated using the following equations.

$$
\begin{aligned}
S_{\text {helix }} & =\left\langle\left(3 \cos ^{2} \beta-1\right) / 2\right\rangle \\
S_{\text {dipole }} & =\left\langle\left(3 \cos ^{2} \alpha-1\right) / 2\right\rangle
\end{aligned}
$$

where $\beta$ is the angle between the transmembrane helix axis and the membrane normal and $\alpha$ is the angle between the principal transition dipole moment and the molecular director. Accordingly, for a transmembrane helix

$$
\begin{aligned}
S_{\text {helix }}=\left[2\left(E_{x}^{2}-R^{\mathrm{ATR}} E_{y}^{2}+E_{z}^{2}\right)\right] / \\
\quad\left[\left(3 \cos ^{2} \alpha-1\right)\left(E_{x}^{2}-R^{\mathrm{ATR}} E_{y}^{2}-2 E_{z}^{2}\right)\right]
\end{aligned}
$$

In the case of the amide I band for the $\alpha$-helix, $\alpha=39^{\circ}$. The helix order parameter, $S_{\text {helix }}$, can then be obtained for the angle $\beta$, the membrane-protein helix tilt angle vis a vis the membrane normal.

Similarly, for the lipid chains

$$
S_{\text {lipids }}=S_{\text {acylchain }} \cdot S_{\text {dipole }}
$$

where $S_{\text {acyl chain }}$ denotes the order parameter associated with acyl chains of the lipid molecules about the membrane normal. $S_{\text {lipids }}$ could be determined from the dichroic ratio $R^{\text {ATR }}$ measured for the bilayer lipids from the parallel and perpendicular intensities of the $v_{\text {as }}\left(\mathrm{CH}_{2}\right)$ band at $2920 \mathrm{~cm}^{-1}$, for which $\alpha=90^{\circ}$. $S_{\text {acyl chain }}$ could then be determined from the measured $S_{\text {lipids }}$ and eq 6 .

\section{RESULTS}

Isolation of $p M M O$. Under the standardized bacterial growth conditions developed in this laboratory, it is possible to obtain overproduction of highly enriched pMMO-containing membranes $(18,19)$. It has recently been shown that pMMO is a multicopper-containing $\alpha \beta \gamma$-protein monomer (19). The sodium dodecyl sulfate-polyacrylamide gel electrophoresis (SDS-PAGE) analysis of the as-isolated pMMO membranes (Figure 1, lane 2) confirms the presence of the three subunits of the pMMO hydroxylase, $45(\alpha), 27(\beta)$, $23(\gamma) \mathrm{kDa}$, as the major fractions. In earlier work (18), it was suggested that the $\sim 35 \mathrm{kDa}$ polypeptide might correspond to a proteolytic fragment or a posttranslational modification of the $45 \mathrm{kDa}$ subunit of pMMO. As evident from Figure 1, in the as-isolated membranes, pMMO accounts for more than $80 \%$ of the total membrane proteins (analysis not shown; see 19).

To study the structure and dynamics of a membrane protein in its native form, the ideal way would be to examine the protein in question embedded in the intact biological membrane. However, the presence of other membrane

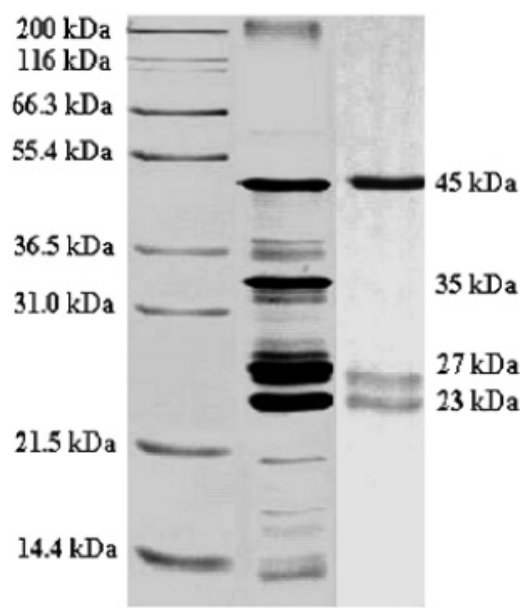

FIGURE 1: SDS-PAGE protein gels. Lane 1, standards; lane 2, pMMO-enriched membranes isolated from $M$. capsulatus (Bath); lane 3, purified pMMO $(0.5 \mathrm{mg} / \mathrm{mL})$ from detergent-solubilized pMMO-enriched membranes (detergent $=$ dodecyl $\beta$-D-maltoside).

proteins limits this approach. Purification and reconstitution of the membrane protein require its solubilization, which could alter its native state. pMMO requires a lipid environment to support its function. Previous attempts at isolating the protein indicated that once it is removed from the lipid bilayer, the enzyme deactivates quickly (18). Although the Chan laboratory has recently obtained a purified preparation of the enzyme by solubilizing the pMMO-enriched membranes in dodecyl $\beta$-D-maltoside and fractionating the protein-detergent micelles by size exclusion chromatography (19), efforts to reconstitute the protein with the full complement of copper ions and high specific activity in lipid membranes have still not met with complete success. Thus, at this juncture, the isolation of pMMO-enriched or overproduced membranes offers the only, if not unique, opportunity to study the membrane protein in its native environment. Accordingly, we have resorted to pMMOenriched membranes for the bulk of the structural analysis described here.

ATR-FTIR Spectrum of pMMO-Enriched Membranes. The unpolarized ATR-FTIR spectra recorded for thin films of the pMMO-enriched membranes in $\mathrm{H}_{2} \mathrm{O}$ buffer and the isolated membrane lipids are compared in Figure 2. The spectrum of the pMMO-enriched membranes showed contributions from the proteins along with the membrane lipids. The two distinct protein-associated peaks observed were at 1656 (amide I) and 1542 (amide II) $\mathrm{cm}^{-1}$. The peak centered at $1656 \mathrm{~cm}^{-1}$ is indicative of the $\alpha$-helix; however, this amide I feature is accompanied by shoulders appearing around 1641 and $1680 \mathrm{~cm}^{-1}$ suggesting substantive contributions also from random coils and $\beta$-sheets, respectively. The ATR-FTIR spectrum of the purified pMMO in a dodecyl $\beta$-D-maltoside detergent micelle was found to be identical to that of the as-isolated membranes in this region (inset of Figure 2), indicating that the spectral contributions from other membrane proteins to the amide I and II regions were negligible in the pMMO-enriched membranes.

Minor absorption bands observed at 2920 and $2852 \mathrm{~cm}^{-1}$ were lipid-associated, arising from the $v_{\mathrm{as}}\left(\mathrm{CH}_{2}\right)$ and $v_{\mathrm{s}}\left(\mathrm{CH}_{2}\right)$, respectively. The lipid ester peak at $1739 \mathrm{~cm}^{-1}$ due to $v(\mathrm{C}=$ $\mathrm{O}$ ) appeared with a very low intensity in the pMMO-enriched membranes. 


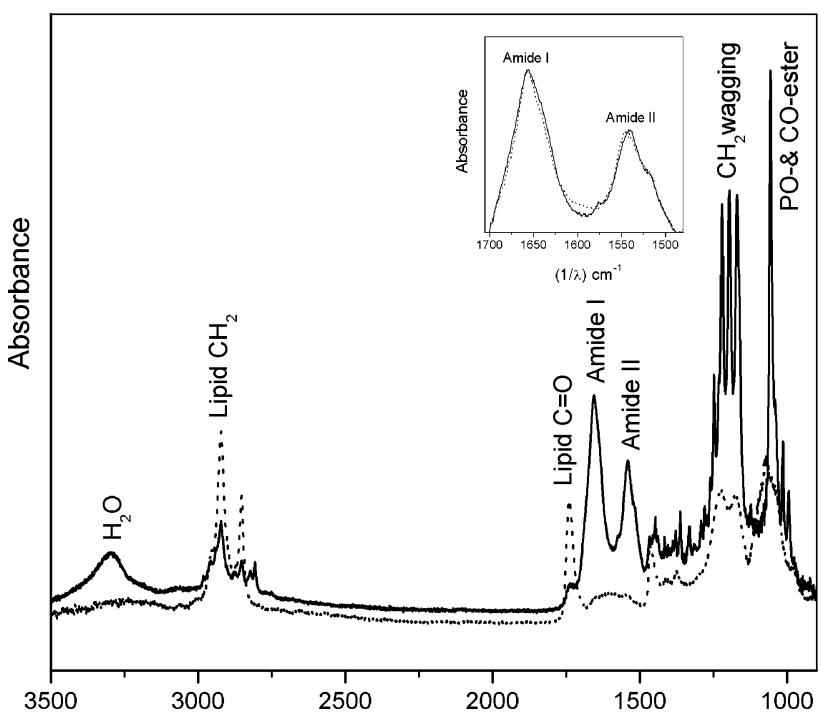

$(1 / \lambda) \mathrm{cm}^{-1}$

FIGURE 2: ATR-FTIR spectra of a thin film of the pMMO-enriched membranes in $\mathrm{H}_{2} \mathrm{O}$ buffer ( - ) and isolated pMMO membrane lipid multilayers $(\cdots)$ spread over germanium surface. Inset: Amide region in the corresponding spectra for a thin pMMO-enriched membrane film ( - ) and purified pMMO in a dodecyl $\beta$-D-maltoside detergent micelle $(\cdots)$ in $\mathrm{H}_{2} \mathrm{O}$ buffer. Note that the $\mathrm{CH}_{2}$ wagging region around $1200 \mathrm{~cm}^{-1}$ includes interference from a number of sharp features from the PIPES buffer, in addition to the contributions from the membrane lipids.

Secondary Structure of $p M M O$. The secondary structure elements of pMMO were obtained from the shape of the amide I peak. Fourier self-deconvolution (GRAMS/386 software) was applied using a Lorentzian line shape [full width at half-height (fwhh) $=25 \mathrm{~cm}^{-1}$ ] to narrow the different components of the amide I region. Prior to the nonlinear curve fitting, a straight baseline passing through the ordinates at 1700 and $1600 \mathrm{~cm}^{-1}$ was subtracted, as noted earlier. The peak positions, obtained from the second derivative of the spectrum, were used as the input parameters for the curve fitting. A nonlinear least squares iterative curve fitting (Origin 7.0 software) was then performed with Gaussian line shapes. The best fit was judged by the $\chi^{2}\left(10^{-6}\right)$ value obtained between the simulated and the experimental spectra. To avoid artifacts due to the self-deconvolution procedure, the fitting was also performed on the original amide I peak (Figure 3).

After the curve fitting, each band was assigned to a secondary structure according to its frequency maximum. The area of each band assigned to a given secondary structure was divided by the molar absorptivity expected for the secondary structure, and the resulting area was then divided by the total area to derive the proportion of the particular secondary structure $(30,40)$. From this analysis, the contributions of the various structural components in the pMMO were obtained. These results are summarized in Table 1.

The principal conclusion to emerge from this analysis is that $\alpha$-helices account for $59 \pm 1 \%$ of the structural components (ca. 552 amino acid residues), depending on the set of molar absorptivities used to derive the contributions from the various secondary structural elements $(41,42)$ (compare columns a and $\mathrm{b}$ in Table 1). Essentially the same $\alpha$-helix content (58\%) was obtained had the peak areas been used to estimate the relative contributions of the various

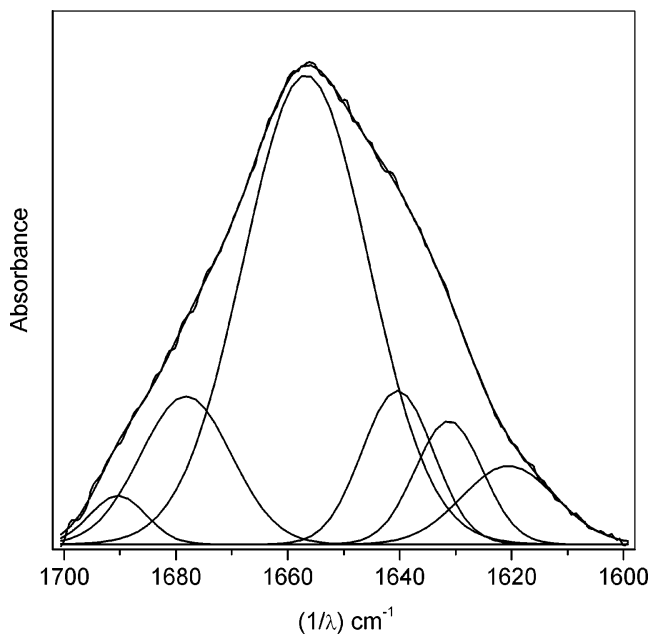

FIGURE 3: pMMO amide I peak along with the results of the nonlinear curve fitting analysis.

\begin{tabular}{clrrr}
\hline Table 1: Secondary Structure Characterization of pMMO \\
\hline \multirow{2}{*}{$\begin{array}{c}\text { frequency of amide-I } \\
\text { component }\left(\mathrm{cm}^{-1}\right)\end{array}$} & $\begin{array}{c}\text { secondary structure } \\
\text { assignment }\end{array}$ & \multicolumn{4}{c}{ peak fraction } \\
\cline { 2 - 5 }$(\%)^{a}$ & $(\%)^{b}$ & $(\%)^{c}$ \\
\hline 1690.4 & $\uparrow \downarrow \beta$-sheet & 1.9 & 1.8 & 2.7 \\
1678.2 & $\uparrow \downarrow \beta$-sheet & 9.2 & 8.7 & 13.2 \\
1656.7 & $\alpha$-helix & 58.2 & 60.1 & 58.0 \\
1641.4 & random coil & 20.0 & 19.3 & 11.0 \\
1632.2 & $\beta$-sheet & 5.7 & 5.4 & 8.2 \\
1620.5 & side chains or & 5.0 & 4.8 & 7.2 \\
& intermolecular $\beta$-sheet & & & \\
\hline
\end{tabular}

${ }^{a}$ Values obtained using molar absorptivities reported by de Jongh et al. (41). ${ }^{b}$ Values obtained using molar absorptivities reported by Kabsch and Sander (42). ${ }^{c}$ Values obtained without correcting for the different molar absorptivities.

secondary structures without correcting for the different molar absorptivities associated with the different structural components (column c in Table 1).

Another conclusion from this analysis of the amide I band was that $\beta$-sheets accounted for $16 \%$ of the secondary structure and random coil as much as $20 \%$. On the other hand, the amino acid side chain contribution (43) to the amide I peak was negligible, as expected. The asparagine, glutamine, arginine, and lysine contents totaled $<3 \%$ from the amino acid composition of pMMO. Finally, although these results were obtained from analysis of the amide I band for the thin sample film, the same conclusions were derived for the thick sample film.

To accommodate hydrogen bonding of the peptide backbone in a hydrophobic environment, integral membrane proteins generally span lipid bilayers in regular secondary structures. The transmembrane $\alpha$-helix is generally the main motif in many membrane proteins, where the interactions between the helices serve to stabilize the protein tertiary structure. As a membrane protein, pMMO is expected to comprise mainly transmembrane helices in the membrane domain. In fact, amino acid sequence analysis of the pMMO subunits using the program TMHMM (TransMembrane topology with a Hidden Markov Model) (44) predicted 15 putative transmembrane $\alpha$-helices. These secondary structure predictions are consistent with the $\alpha$-helical content estimated from the analysis of the amide I band. Thus, it is evident that $\mathrm{pMMO}$ is indeed an $\alpha$-helical bundle embedded in the membrane. 


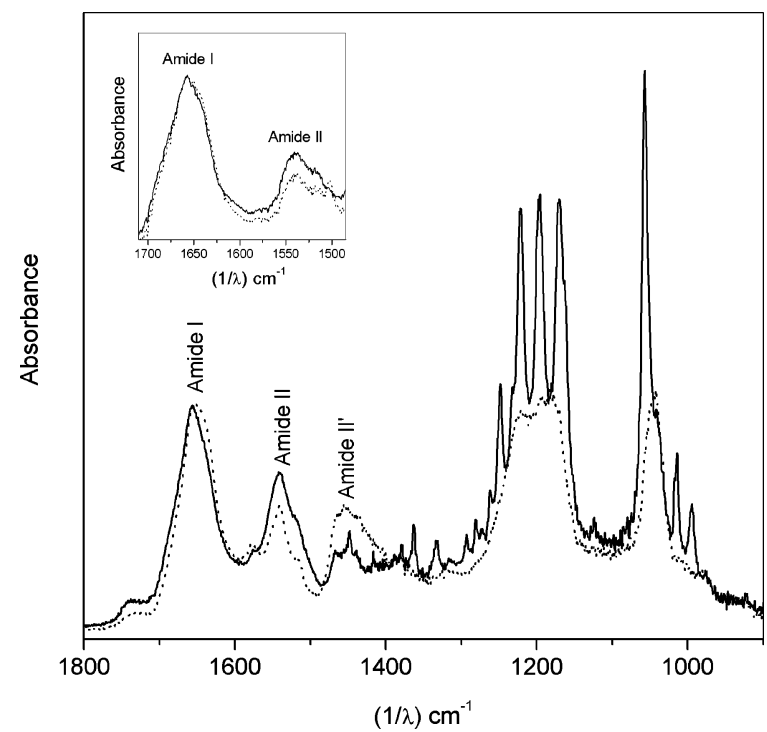

FIGURE 4: ATR-FTIR spectra of a thick pMMO-enriched membrane film before (-) and after ( $\cdots) 21 \mathrm{~h}$ of deuteration. Inset: Amide region in the corresponding spectrum for a thin pMMOenriched membrane film.

ATR-FTIR Spectrum of pMMO-Enriched Membranes after ${ }^{1} H /{ }^{2} H$ Exchange. In Figure 4, the spectra recorded before and after deuteration of both the thin and the thick pMMO membrane films are compared. The measurements on the thick film were taken here as the primary data as the $\mathrm{S} / \mathrm{N}$ ratio of the ATR-FTIR spectrum was significantly better than that obtained for the thin membrane film as expected (see the inset of Figure 4 for data on the thin film). Upon deuteration, a shift in the amide I region from 1656 to 1652 $\mathrm{cm}^{-1}$ along with a decrease of the amide II intensity near $1542 \mathrm{~cm}^{-1}$ and the appearance of amide II' peak in the region $1500-1450 \mathrm{~cm}^{-1}$ (Figure 4) were noted.

While the amide I peak at $1656 \mathrm{~cm}^{-1}$ is typically characteristic of an $\alpha$-helix, peptides in a solvent-exposed "random coil" conformation could also contribute to the region around $1656 \mathrm{~cm}^{-1}$ in ${ }^{1} \mathrm{H}_{2} \mathrm{O}$. However, the extent of the amide I shift toward smaller wavenumbers upon ${ }^{1} \mathrm{H} /{ }^{2} \mathrm{H}$ exchange is characteristic of the type of secondary structure. The random coil peptides normally show much higher shifts $\left(\sim 12 \mathrm{~cm}^{-1}\right)$ as compared to the shifts $\left(<5 \mathrm{~cm}^{-1}\right)$ observed for helical structures (45-47). The absorption feature at 1656 $\mathrm{cm}^{-1}$ observed for $\mathrm{pMMO}$ in ${ }^{1} \mathrm{H}_{2} \mathrm{O}$ shifted to $1652 \mathrm{~cm}^{-1}$ upon ${ }^{1} \mathrm{H} /{ }^{2} \mathrm{H}$ exchange, confirming that it arises from the $\alpha$-helical secondary structure.

The amide II peak intensity near $1542 \mathrm{~cm}^{-1}$ is reduced upon ${ }^{1} \mathrm{H} /{ }^{2} \mathrm{H}$ exchange as the in-plane $\mathrm{N}-{ }^{2} \mathrm{H}$ bending vibrations appear in the $1040-940 \mathrm{~cm}^{-1}$ region while the $v(\mathrm{C}-$ $\mathrm{N})$ vibration moved to the $1490-1450 \mathrm{~cm}^{-1}$ region mixing with other modes to yield a new band designated as amide II'. Accordingly, reduction in the amide II absorption intensity can be used to measure the extent of amide ${ }^{1} \mathrm{H} /{ }^{2} \mathrm{H}$ exchange. This provides a reliable indication of the extent of protection of the protein toward amide ${ }^{1} \mathrm{H} /{ }^{2} \mathrm{H}$ exchange due to tertiary folding, including the penetration of the polypeptides into the membrane (48). If the amide II peak area between 1596 and $1502 \mathrm{~cm}^{-1}$ before deuteration was taken as $100 \%$ unexchanged protein, total deuteration of the protein would yield zero absorption intensity in this region. From a comparison of the spectral intensity in this region

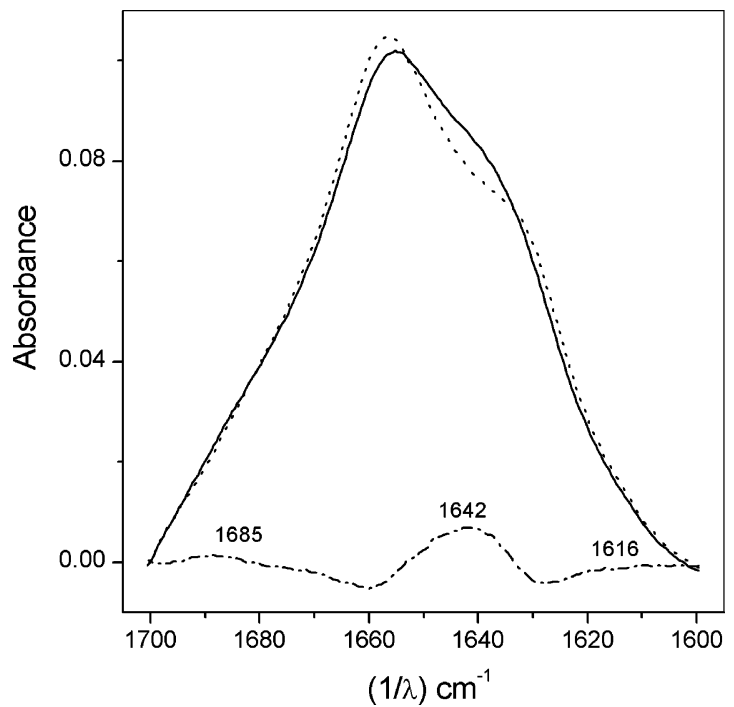

FIGURE 5: Amide I peak region in the ATR-FTIR spectra of the intact ( - ) and proteolyzed ( $\cdots$ ) pMMO in $\mathrm{H}_{2} \mathrm{O}$ buffer along with their difference spectrum (- - ).

before and after ${ }^{1} \mathrm{H} /{ }^{2} \mathrm{H}$ exchange, about $80 \%$ of the peptide residues of pMMO (ca. 740 amino acids) were estimated to be protected from the amide ${ }^{1} \mathrm{H} /{ }^{2} \mathrm{H}$ exchange during a $21 \mathrm{~h}$ exposure to $98 \%{ }^{2} \mathrm{H}_{2} \mathrm{O}$ relative humidity (for both the thin and the thick films). This result indicates that a significant portion of the protein is buried in the hydrophobic bilayer and that much of the aqueous exposed domains of the pMMO are also protected from amide ${ }^{1} \mathrm{H} /{ }^{2} \mathrm{H}$ exchange. This result is corroborated by the solvent accessibility composition (core/ surface ratio) predicted for the pMMO by using the software PROF (PROFile network prediction HeiDelberg) (49). In this analysis, $70 \%$ of the polypeptide residues were judged to be totally buried, while the remaining $30 \%$ were considered to be exposed with greater than $16 \%$ of their surfaces accessible to the solvent.

ATR-FTIR Spectra of Proteolyzed pMMO-Enriched Membranes. The extent of the amide hydrogen exchange by deuterium is related to the stability of the secondary structures and the solvent accessibility to the amide groups. Amide proton ${ }^{1} \mathrm{H} /{ }^{2} \mathrm{H}$ exchange occurs slowly within the membrane bilayer since this process would require disruption of the backbone hydrogen-bonding pattern and exposure of the highly polar peptide bonds in the extremely hydrophobic environment $(50,51)$. To confirm that the transmembrane domains of pMMO form an $\alpha$-helical bundle embedded in a bilayer, we carried out proteolysis of the protein with trypsin. With this procedure, we have potentially isolated and removed the cytoplasmic domain of the pMMO, leaving intact only the membrane domains. Figure 5 depicts the amide I peak region of the ATR-FTIR spectra of the intact and proteolyzed (in situ membrane trypsin proteolysis carried out prior to film formation) protein along with the difference spectrum. The spectral feature corresponding to $\alpha$-helical structure in the amide I region for the membrane fraction after proteolysis was observed to be essentially the same as for the intact protein. The amide I difference spectrum showed a peak at $1642 \mathrm{~cm}^{-1}$ with shoulders around 1680 and $1616 \mathrm{~cm}^{-1}$, but essentially a "null" was observed at 1656 $\mathrm{cm}^{-1}$, where the amide I band is expected to peak for $\alpha$-helices. 
Table 2: Amino Acid Sequence of the $45 \mathrm{kDa}$ Subunit (PmoB) of pMMO from M. capsulatus (Bath) with the Transmembrane Helices Predicted by TMHMM Depicted in Red and the Regions Predicted to Be External to the Lipid Membrane Shown in Bold Letters ${ }^{a}$

$\begin{array}{lllll}\text { MKTIKDRIAK }^{10} & \text { WSAIGLLSAV }^{20} & \text { AATAFYAPSA }^{30} & \text { SAHGEKSQAA }^{40} & \text { FMRMRTIHWY }^{50} \\ \text { DLSWSKEKVK }^{60} & \text { INETVEIKGK }^{70} & \text { FHVFEGWPET }^{80} & \text { VDEPDVAFLN }^{90} & \text { VGMPGPVFIR }^{100} \\ \text { KESYIGGQLV }^{100} & \text { PRSVRLEIGK }^{120} & \text { TYDFRVVLKA }^{130} & \text { RRPGDWHVHT }^{100} & \text { MMNVQGGGPI }^{150} \\ \text { IGPGKWITVE }^{100} & \text { GSMSEFRNPV }^{170} & \text { TTLTGQTVDL }^{100} & \text { ENYNEGNTYF }^{190} & \text { WHAFWFAIGV }^{200} \\ \text { AWIGYWSRRP }^{210} & \text { IFIPRLLMVD }^{20} & \text { AGRADELVSA }^{200} & \text { TDRKVAMGFL }^{200} & \text { AATILIVVMA }^{250} \\ \text { MSSANSKYPI }^{200} & \text { TIPLQAGTMR }^{200} & \text { GMKPLELPAP }^{20} & \text { TVSVKVEDAT }^{20} & \text { YRVPGRAMRM }^{300} \\ \text { KLTITNHGNS }^{310} & \text { PIRLGEFYTA }^{320} & \text { SVRFLDSDVY }^{300} & \text { KDTTGYPEDL }^{340} & \text { LAEDGLSVSD }^{350} \\ \text { NSPLAPGETR }^{300} & \text { TVDVTASDAA }^{300} & \text { WEVYRLSDII }^{300} & \text { YDPDSRFAGL }^{300} & \text { LFFFDATGNR }^{400} \\ \text { QVVQIDAPLI }^{40} & \text { PSFM }^{414} & & & \end{array}$

${ }^{a}$ The tryptic peptides identified by mass spectroscopy are highlighted in yellow.

It has been proposed earlier that among the three subunits [45 $(\alpha), 27(\beta)$, and $23(\gamma) \mathrm{kDa}$ ] of the pMMO hydroxylase, part of the $45 \mathrm{kDa}$ subunit is solvent exposed, hydrophilic, and contains the E-clusters $(17,18)$. Peptide mass fingerprinting and the amino acid sequence coverage of the tryptic peptides showed that they all originated from the $45 \mathrm{kDa}$ subunit of the pMMO hydroxylase.

According to TMHMM topology predictions (44), the identified tryptic peptides are located in the region of the polypeptide external to the lipid membrane (Table 2). TMHMM and PROF secondary structure predictions for this subunit yielded three transmembrane helices while the parts associated with the cytoplasmic/periplasmic domains were predicted to be mostly $\beta$-sheet. Consistent with these predictions, the difference spectrum of the intact and the proteolyzed pMMO membranes showed absorption peaks only in the spectral regions characteristic for unordered and $\beta$-structures (Figure 5). It is evident from this experiment that the protein helices are indeed membrane-embedded.

Comparison of the ATR-FTIR Spectra of As-Isolated, Fully Reduced, and Fully Oxidized pMMO in pMMO-Enriched Membranes. Up to this point, all of the spectroscopic observations have been made on pMMO-enriched membranes as isolated from the harvested cells. Under these conditions, the C-cluster copper ions, which participate directly in the dioxygen and hydroxylation chemistry of the enzyme, are oxidized, while the E-cluster copper ions remain fully reduced $(7,20)$. Earlier studies $(17,18)$ have predicted that the pMMO catalytic sites containing the metallic core (C-clusters) are embedded in the membrane. As the dioxygen is activated at the metal cofactor(s) for transfer of one of the oxygen atoms to the substrate, the substrate-binding site must also be close to the cofactor. Protein breathing and collective motions should also be activated upon binding of the dioxygen and lead to conformational changes in the protein. These changes should be reflected in the conformation sensitive amide I band. Toward this end, we have compared the ATR-FTIR spectrum of the as-isolated pMMO with the spectra of the fully reduced and oxidized states in

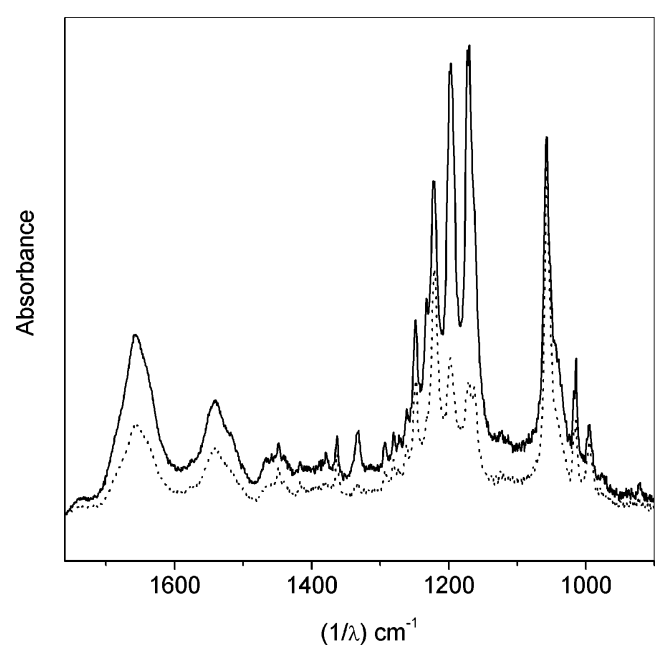

FIGURE 6: ATR-FTIR spectra of a thin pMMO-enriched membrane film recorded with incident light polarized parallel $(-)$ or perpendicular $(\cdots)$ to the plane of incidence.

the pMMO-enriched membranes. However, only a small shift in the absorption maxima of the amide I peak was noticed between the different redox states of the enzyme (data not shown).

Orientation of the pMMO Secondary Structure. Determination of the orientation of an $\alpha$-helix in a membrane protein with respect to the lipid bilayer, or the so-called $\alpha$-helix tilt angle, requires the evaluation of the dichroic ratio $R^{\mathrm{ATR}}$ for a vibrational band associated with the helix, which is obtained from the polarized spectra recorded with the electric field vector of the IR radiation polarized parallel or perpendicular to the incidence plane $(39,52)$. Similarly, the dichroic ratio $R^{\text {ATR }}$ for a vibrational band associated with the acyl chain of a lipid molecule allows the determination of the mean or most probable tilt angle of the lipid acyl chains with respect to the membrane normal.

Polarized spectra recorded for the proteins in the pMMOenriched membranes in $\mathrm{H}_{2} \mathrm{O}$ buffer are shown in Figure 6. To determine the orientation of the hydrocarbon chains in the bilayer alone, the polarized spectra for the lipids isolated 
from pMMO membranes were also recorded. Strong positive dichroism was observed for both the proteins and the lipids in the pMMO-enriched membranes (Figure 6) as well as for the isolated lipids (spectra not shown). The dichroic ratio $R^{\mathrm{ATR}}$ observed for $v_{\text {as }}\left(\mathrm{CH}_{2}\right)$ at $2920 \mathrm{~cm}^{-1}$ of the bilayer lipids in the pMMO-enriched membranes or for the bacterial lipids isolated from the membranes was $1.5 \pm 0.04$. This outcome is significant, as we have obtained the same dynamic structure for the bilayer lipids in the pMMO-enriched membranes as well as for the bilayer membranes formed from bacteria lipids isolated from the cell membranes of the organism.

The dichroic ratio measured for the lipid molecule represents the average $R^{\mathrm{ATR}}$ for all of the $\mathrm{CH}_{2}$ segments along the two hydrocarbon chains. When this $R^{\text {ATR }}$ value was substituted into eq 6 , a value of $0.2 \pm 0.03$ was obtained for the order parameter $S_{\text {acylchain, }}$ if it is assumed that the bulk of hydrocarbon chains is in the all-trans configuration, for which $\alpha=90^{\circ}$. The average $\alpha$ for a typical $\mathrm{CH}_{2}$ segment is necessarily less than $90^{\circ}$ due to gauche-trans isomerization along the chain, so the deduced value for $S_{\text {acyl chain }}$ is clearly a lower limit. For a bilayer of saturated lipids above the thermal phase transition, $S_{\text {acyl chain }}$ is typically of the order of $0.4-0.5$, as determined by NMR methods (53).

The fully reduced, as-isolated, and fully oxidized pMMOs correspond to three states of the enzyme with increasing oxidizing equivalents. As noted earlier, in the as-isolated enzyme, only the catalytic copper clusters are oxidized (Cclusters) while the copper ions that mediate electron transfer from NADH (E-clusters) remain reduced $(17,18)$. The average order parameter of the protein helices was determined from the dichroic ratio measured for the amide I vibration in the region $1662-1645 \mathrm{~cm}^{-1}$ for each of the three redox states of the enzyme. For the fully reduced enzyme in the pMMO-enriched membranes, $R^{\mathrm{ATR}}=2.21 \pm 0.02$, and using eq 5, we obtained a value of $0.42 \pm 0.02$ for the average helix order parameter $\left\langle S_{\text {helix }}\right\rangle$ in the fully reduced enzyme. From these polarized ATR-FTIR measurements on pMMO-enriched membranes, we find that the dichroic ratio $R^{\text {ATR }}$ decreases from $2.21 \pm 0.02$ in the case of the fully reduced protein to the value of $1.86 \pm 0.03$ for the as-isolated pMMO. The corresponding dichroic ratio for the fully oxidized enzyme $(2.10 \pm 0.03)$, however, is only slightly smaller than the value observed for the fully reduced enzyme in these same membranes. From these data, we find that the average order parameter for the transmembrane helices in the membranes $\left(\left\langle S_{\text {helix }}\right\rangle\right)$ decreases from a value of $0.42 \pm$ 0.02 in the fully reduced pMMO to $0.14 \pm 0.03$ when the C-cluster copper ions are oxidized, but it returns to the higher value of $0.34 \pm 0.03$ when all of the copper ions are oxidized. It is evident that there is a substantial change in the overall orientational order of the transmembrane $\alpha$-helices about the membrane normal among the different redox states of the enzyme, particularly, when the C-cluster copper ions are oxidized. In other words, there is a significant adjustment in the packing of the transmembrane $\alpha$-helices upon dioxygen activation of the enzyme.

We have compared the protein helix order parameters for the pMMO in thin and thick films of the pMMO-enriched membranes. Not surprisingly, the measured average helix order parameter was substantially smaller for the thick membrane film: $\left\langle S_{\text {helix }}\right\rangle=0.14 \pm 0.01$ in the case of the

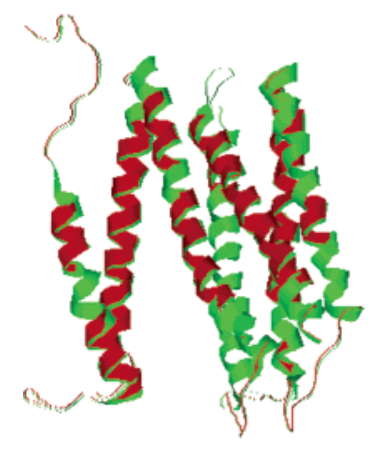

a

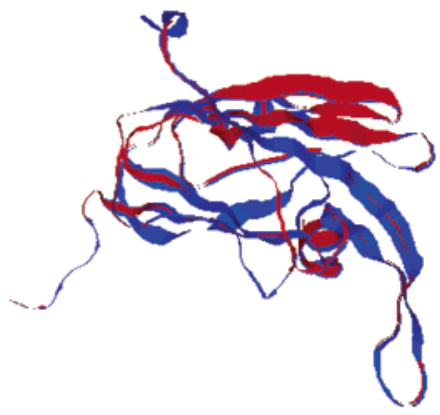

b
FIGURE 7: Three-dimensional PSSM results for the pMMO subunits. (a) Superimposition of the pMMO $\gamma$-subunit model (red) over the template (green). (b) Superimposition of the pMMO $\alpha$-subunit (N255-M414) model (red) over the template (blue).

fully reduced pMMO, implicating a sizable domain spread of the membrane normals in the thick membrane. Our thick film is a multilayer of some 200 bilayer membranes, and accordingly, not all of the cooperative domains can be expected to be perfectly aligned. In other words, there is a distribution of membrane normals relative to the IRE for the individual cooperative membrane domains. In contrast, our thin pMMO-enriched membrane film consists of a stack of only four or five bilayers.

Three-Dimensional Fold Recognition of pMMO. Finally, we summarize some preliminary results of attempts to probe the three-dimensional folding pattern of the pMMO subunits by computational methods. For this analysis, we have used the on line fold recognition server 3D PSSM (http:// www.sbg.bio.ic.ac.uk/3dpssm). Three-dimensional PSSM uses a three-dimensional position specific scoring matrix to recognize remote protein sequence homologues (54). The method combines the power of multiple sequence profiles with the knowledge of protein structure to provide enhanced recognition and thus functional assignment of newly sequenced genomes. The method uses structural alignments of homologous proteins of similar three-dimensional structure in the structural classification of proteins (SCOP) database to obtain a structural equivalence of residues. These equivalences are used to extend multiply aligned sequences obtained by standard sequence searches. The resulting large superfamily-based multiple alignment is converted into a PSSM. The reliability of the results from this server depends on a "PSSM $E$-value", which is the score or expectation value of the match representing the " $\%$ of certainty" between the query and the template.

In the case of the pMMO, it was possible to obtain a 80$90 \%$ match for the sequences defining the $\gamma$-subunit and part of the solvent-exposed region of the $\alpha$-subunit. Although the sequence identity between the query and the template is no more than $20 \%$, the three-dimensional fold pattern between them is very similar. It is interesting that the best match $\left(E_{\mathrm{PSSM}}=0.207\right)$ for the $\gamma$-subunit is a fold (Figure 7a) coming from the protein cytochrome $c$ oxidase in Paracoccus dentrificans (A1-R273 of PDB entry 1qle). This protein belongs to a membrane all- $\alpha$ protein family, while for the C-terminal solvent-exposed sequence (N255-M414) of the pMMO $\alpha$-subunit, the best match $\left(E_{\mathrm{PSSM}}=0.155\right)$ is a fold (Figure $7 \mathrm{~b}$ ) coming from the protein nitrite reductase in Acromobacter cyloclastes (D8-K166 of PDB entry 1nif). 
This protein belongs to a class of all- $\beta$ proteins and comes from the family of multidomain cupredoxins. Our experimental ATR-FTIR data offer strong support to these secondary structure and topology predictions.

\section{DISCUSSION}

The principal conclusion to emerge from the present ATRFTIR study of pMMO is that the protein is an $\alpha$-helical bundle with ca. 15 transmembrane $\alpha$-helices embedded in the bilayer membrane, together with a water-exposed domain comprised mostly of a $\beta$-sheet typical of a class of all- $\beta$ proteins belonging to the family of multidomain cupredoxins. The C-cluster copper ions are thought to be associated with the transmembrane domain, and presumably, the E-cluster copper ions are part of the $\beta$-sheet domain. Second, the protein helices are embedded in the membrane bilayer with an overall average orientational order parameter for the helices about the membrane normal of $\left\langle S_{\text {helix }}\right\rangle=0.42 \pm 0.02$ in the case of the fully reduced protein. There is a significant change in the packing order of the transmembrane $\alpha$-helices upon dioxygen activation of the enzyme. Although the above conclusions were derived from ATR-FTIR measurements on pMMO-enriched membranes $80 \%$ enriched in this protein, similar results were obtained from measurements on purified pMMO in a dodecyl $\beta$-D-maltoside detergent micelle reconstituted in dimyristoylphosphatidylcholine (DMPC) lipids. In any case, only a small number of other membrane proteins, e.g., the oxidases, aside from the pMMO, are expected to be sensitive to molecular oxygen, and these are present in minute amounts in our pMMO-enriched membranes.

It has been reported (21) that the packing of the $\alpha$-helices, or the relative tilt of $\alpha$-helices forming the membrane domain of a protein embedded in a bilayer, is less ordered in cofactorbinding proteins. This phenomenon may be required by cavity formation or the splaying of the helices to generate a pocket to accommodate the cofactor. Such a cavity has been noted around the iron center in the case of sMMO (14). The two iron atoms and the coordinating ligands are located on one internal surface of this cavity, while the rest of the cavity is filled with the hydrophobic side chains. This sort of splaying of the $\alpha$-helices may be present in the hydrophobic membrane-embedded domains of pMMO in order to create a cavity to accommodate the catalytic site.

The extraordinary chemistry catalyzed by pMMO has no precedent in known model and biological systems. The ability to bind and hydroxylate a substrate as small and nonpolar as methane is one of the most intriguing properties of this enzyme. Characterizing the structure of the membraneembedded domain of pMMO is of major importance for the understanding of its molecular mechanism. Although the three-dimensional folding of this enzyme would provide important insight into the mechanism of biological methane oxidation, including how these inert gases might diffuse to and bind near the active site, until the three-dimensional structure of pMMO is solved, the ATR-FTIR results presented here must provide a preliminary structural framework for understanding the structure-reactivity pattern of this important enzyme.

\section{REFERENCES}

1. Higgins, I. J., Best, D. J., and Hammond, R. C. (1980) New findings in methane-utilizing bacteria highlight their importance in the biosphere and their commercial potential, Nature 286, 561564.

2. Pearman, G. I., and Fraser, P. J. (1988) Sources of increased methane, Nature 332, 489-490.

3. Anthony, C. (1982) The Biochemistry of Methylotrophs, Academic Press, London.

4. Bédard, C., and Knowles, R. (1989) Physiology biochemistry, and specific inhibitors of methane, ammonia, and carbon monoxide oxidation by methanotrophs and nitrifiers, Microbiol. Rev. 53, 6884.

5. Crabtree, R. H. (1995) Aspects of methane chemistry, Chem. Rev. 95, 987-1007.

6. Lipscomb, J. D. (1994) Biochemistry of the soluble methane monooxygenase, Annu. Rev. Microbiol. 48, 371-399.

7. Nguyen, H.-H. T., Nakagawa, K. H., Hedman, B., Elliott, S. J., Lidstrom, M. E., Hodgson, K. O., and Chan, S. I. (1996) X-ray Absorption and EPR studies on the copper ions associated with the particulate methane monooxygenase from Methylococcus capsulatus (Bath). $\mathrm{Cu}(\mathrm{I})$ ions and their implications, J. Am. Chem. Soc. 118, 12766-12776.

8. Stirling, D. I., and Dalton, H. (1980) Oxidation of dimethyl ether, methyl formate, and bromomethane by Methylococcus capsulatus (Bath), J. Gen. Microbiol. 116, 277-283.

9. Green, J., and Dalton, H. (1989) Substrate specificity of soluble methane monooxygenase. Mechanistic implications, J. Biol. Chem. $264,17698-17703$.

10. Huang, D.-S., Wu, Y.-S., Yu, S. S.-F., and Chan, S. I. (2002) Determination of the carbon kinetic isotope effects on propane hydroxylation mediated by the methane monooxygenases from Methylococcus capsulatus (Bath) by using stable carbon isotope analysis, ChemBioChem. 3, 760-765.

11. Dewitt, J. G., Bentsen, J. G., Rosenzweig, A. C., Hedman, B., Green, J., Pilkington, S., Papaefthymiou, G. C., Dalton, H., Hodgson, K. O., and Lippard, S. J. (1991) X-ray absorption, Mössbauer, and EPR studies of the dinuclear iron center in the hydroxylase component of methane monooxygenase, J. Am. Chem. Soc. 113, 9219-9235.

12. Pulver, S., Froland, W. A., Fox, B. G., Lipscomb, J. D., and Solomon, E. I. (1993) Spectroscopic studies of the coupled binuclear non-heme iron active site in the fully reduced hydroxylase component of methane monooxygenase: Comparison to deoxy and deoxy-azide hemerythrin, J. Am. Chem. Soc. 115, $12409-12422$.

13. Liu, K. E., Valentine, A. M., Wang, D. L., Huynh, B. H., Edmondson, D. E., Salifoglou, A., and Lippard, S. J. (1995) Kinetic and spectroscopic characterization of intermediates and component interactions in reactions of methane monooxygenase from Methylococcus capsulatus (Bath), J. Am. Chem. Soc. 117, 10174-10185.

14. Rosenzweig, A. C., Frederick, C. A., Lippard, S. J., and Nordlund, P. (1993) Crystal structure of a bacterial non-heme iron hydroxylase that catalyzes the biological oxidation of methane, Nature $366,537-543$.

15. Elango, N., Radhakrishnan, R., Froland, W. A., Jr., Wallar, B. J., Earhart, C. A., Lipscomb, D., and Ohlendorf, D. H. (1997) Crystal structure of the hydroxylase component of methane monooxygenase from Methylosinus trichosporium OB3b, Protein Sci. 6, 556568.

16. Chan, S. I., Nguyen, H.-H. T., Shiemke, A. K., and Lidstrom, M. E. (1993) Bioinorganic Chemistry of Copper (Karlin, K. D., and Tyeklar, Z., Eds.) pp 184-195, Chapman and Hall, New York.

17. Semrau, J. D., Zolandz, D., Lidstrom, M. E., and Chan, S. I. (1995) The role of copper in the pMMO of Methylococcus capsulatus (Bath): A structural vs catalytic function, J. Inorg. Biochem. 58, $235-244$

18. Nguyen, H.-H. T., Elliott, S. J., Yip, J. K., and Chan, S. I. (1998) The particulate methane monooxygenase from Methylococcus capsulatus (Bath) is a novel copper-containing three-subunit enzyme. Isolation and characterization, J. Biol. Chem. 273, 79577966.

19. Yu, S. S.-F., Chen, K. H.-C., Tseng, Y.-H. M., Wang, Y.-S., Tseng, C.-F., Chen, Y.-J., Huang, D.-S., and Chan, S. I. (2003) Production of high quality pMMO in high yields from Methylococcus capsulatus (Bath) with a hollow-fiber membrane bioreactor, $J$. Bacteriol. 185, 5915-5924.

20. Chan, S. I., Chen, K. H.-C., Tseng, Yu, S. S.-F., Chen, C.-L., and Kuo, S. S.-J. (2004) Toward delineating the structure and function of the particulate methane monooxygenase from methanotrophic bacteria. Biochemistry 43, 4421-4430. 
21. Arkin, I. T., Russ, W. P., Lebendiker, M., and Schuldiner, S. (1996) Determining the secondary structure and orientation of EmrE, a multi-drug transporter, indicates a transmembrane four-helix bundle, Biochemistry 35, 7233-7238.

22. Goormaghtigh, E., Raussens, V., and Ruysschaert, J.-M. (1999) Attenuated total reflection infrared spectroscopy of proteins and lipids in biological membranes, Biochim. Biophys. Acta 1422, $105-185$.

23. Bechinger, B., Ruysschaert, J.-M., and Goormaghtigh, E. (1999) Membrane helix orientation from linear dichroism of infrared attenuated total reflection spectra, Biophys. J. 76, 552-563.

24. Grimard, V., Vigano, C., Margolles, A., Wattiez, R., Veen, H. W. von, Konings, W. N., Ruysschaert, J.-M., and Goormaghtigh, E. (2001) Structure and dynamics of the membrane-embedded domain of LmrA investigated by coupling polarized ATR-FTIR spectroscopy and ${ }^{1} \mathrm{H} /{ }^{2} \mathrm{H}$ exchange, Biochemistry 40, 1187611886.

25. Ding, F., Xie, H., Arshava, B., Becker, J. M., and Naider, F. (2001) ATR-FTIR study of the structure and orientation of transmembrane domains of the Saccharomyces cerevisiae $\alpha$-mating factor receptor in phospholipids, Biochemistry 40, 8945-8954.

26. Rich, P. R., and Breton, J. (2002) Attenuated total reflection Fourier transform infrared studies of redox changes in bovine cytochrome $c$ oxidase: Resolution of the redox Fourier transform infrared difference spectrum of heme $\mathrm{a}_{3}$, Biochemistry 41, 967-973.

27. Talulian, S. A. (2003) Attenuated total reflection Fourier transform infrared spectroscopy: A method of choice for studying membrane proteins and lipids, Biochemistry 42, 11898-11907.

28. Chen, K. H.-C., Chen, C.-L., Tseng, C.-F., Yu, S. S.-F., Ke, S. C., Lee, J.-F., Nguyen, H. T., Elliott, S. J., Alben, J. O., and Chan, S. I. (2004) The copper clusters in the particulate methane monooxygenase (pMMO) from Methylococcus capsulatus (Bath), J. Chin. Chem. Soc. In press.

29. Fringeli, U. P., and Guenthard, H. H. (1981) Infrared membrane spectroscopy, Mol. Biol. Biochem. Biophys. 31, 270-332.

30. Goormaghtigh, E., Cabiaux, V., and Ruysschaert, J.-M. (1990) Secondary structure and dosage of soluble and membrane proteins by attenuated total reflection Fourier transform infrared spectroscopy on hydrated films, Eur. J. Biochem. 193, 409-420.

31. Goormaghtigh, E., Cabiaux, V., and Ruysschaert, J.-M. (1994) Determination of soluble and membrane protein structure by Fourier transform infrared spectroscopy. II. Experimental aspects, side chain structure, and H/D exchange, Subcell. Biochem. 23 , 363-403.

32. Goormaghtigh, E., Cabiaux, V., and Ruysschaert, J.-M. (1994) Determination of soluble and membrane protein structure by Fourier transform infrared spectroscopy. III. Secondary structures, Subcell. Biochem. 23, 405-450.

33. Arrondo, J. L., Etxabe, I., Dornberger, U., and Goni, F. M. (1994) Probing protein conformation by infrared spectroscopy, Biochem. Soc. Trans. 22, 380S.

34. Krimm, S., and Bandekar, J. (1986) Vibrational spectroscopy and conformation of peptides, polypeptides, and proteins, Adv. Protein Chem. 38, 181-364.

35. Susi, H., and Byler, D. M. (1986) Resolution-enhanced Fourier transform infrared spectroscopy of enzymes, Methods Enzymol. $130,290-311$.

36. Harrick, N. J. (1967) Internal Reflection Spectroscopy, Interscience Publisher, New York.

37. Smith, S. O., Jonas, S. R., Braiman, M., and Bormann, B. J. (1994) Structure and orientation of the transmembrane domain of glycophorin A in lipid bilayers, Biochemistry 33, 6334-6341.

38. Axelsen, P. H., and Citra, M. J. (1996) Orientational order determination by internal reflection infrared spectroscopy, Prog. Biophys. Mol. Biol. 66, 227-253.
39. Menikh, A., Saleh, M. T., Gariepy, J., and Boggs, J. M. (1997) Orientation in lipid bilayers of a synthetic peptide representing the C-terminus of the A1 domain of Shiga Toxin. A polarized ATR-FTIR study, Biochemistry 36, 15865-15872.

40. Byler, M. D., and Susi, H. (1986) Examination of the secondary structure of proteins by deconvoluted FTIR spectra, Biopolymers $25,469-487$.

41. de Jongh, H. H. J., Goormaghtigh, E., and Ruysschaert, J.-M. (1996) The different molar absorptivities of the secondary structure types in the amide I region: An attenuated total reflection infrared study on globular proteins, Anal. Biochem. 242, 95-103.

42. Kabsch, W., and Sander, C. (1983) Dictionary of protein secondary structure: Pattern recognition of hydrogen-bonded and geometrical features, Biopolymers 22, 2577-2637.

43. Venyaminov, S. Yu., and Kalnin, N. N. (1990) Quantitative IR spectrophotometry of peptide compounds in water $\left(\mathrm{H}_{2} \mathrm{O}\right)$ solutions. Spectral parameters of amino acid residue absorption bands, Biopolymers 30, 1243-1257.

44. Krogh, A., Larsson, B., Heijne, G. von, and Sonnhammer, E. L. L. (2001) Predicting transmembrane protein topology with a hidden Markov model: Application to complete genomes, J. Mol. Biol. 305, 567-580.

45. Tamm, L. K., and Tatulian, S. A. (1993) Orientation of functional and nonfunctional PTS permease signal sequences in lipid bilayers. A polarized attenuated total reflection infrared study, Biochemistry $32,7720-7726$

46. Baenziger, J. E., and Methot, N. (1995) Fourier transform infrared and hydrogen/deuterium exchange reveal an exchange-resistant core of $\alpha$-helical peptide hydrogens in the nicotinic acetylcholine receptor, J. Biol. Chem. 270, 29129-29137.

47. Corbin, J., Methot, N., Wang, H. H., Baenziger, J. E., and Blanton, M. P. (1998) Secondary structure analysis of individual transmembrane segments of the nicotinic acetylcholine receptor by circular dichroism and Fourier transform infrared spectroscopy, J. Biol. Chem. 273, 771-777.

48. Earnest, T. N., Herzfeld, J., and Rothschild, K. J. (1990) Polarized Fourier transform infrared spectroscopy of bacteriorhodopsin. Transmembrane alpha helices are resistant to hydrogen/deuterium exchange, Biophys. J. 58, 1539-1546.

49. Rost, B., Fariselli, P., and Casadio, R. (1996) Topology prediction for helical transmembrane proteins at $86 \%$ accuracy, Protein Sci. $5,1704-1718$.

50. Holloway, P. W., and Buchheit, C. (1990) Topography of the membrane-binding domain of cytochrome $b_{5}$ in lipids by Fourier transform infrared spectroscopy, Biochemistry 29, 9631-9637.

51. Zhang, Y. P., Lewis, R. N., Hodges, R. S., and McElhaney, R. N (1992) FTIR spectroscopic studies of the conformation and amide hydrogen exchange of a peptide model of the hydrophobic transmembrane $\alpha$-helices of membrane proteins, Biochemistry $31,11572-11578$

52. Sharon, M., Oren, Z., Shai, Y., and Anglister, J. (1999) 2D-NMR and ATR-FTIR study of the structure of a cell-selective diastereomer of melittin and its orientation in phospholipids, Biochemistry 38, 15305-15316.

53. Petersen, N. O., and Chan, S. I. (1977) More on the motional state of lipid bilayer membranes: Interpretation of order parameters obtained from nuclear magnetic resonance experiments, Biochemistry 16, 2657-2667.

54. Kelley, L. A., MacCallum, R. M., and Sternberg, M. J. E. (2000) Enhanced genome annotation using structural profiles in the program 3D-PSSM, J. Mol. Biol. 299, 499-520. 